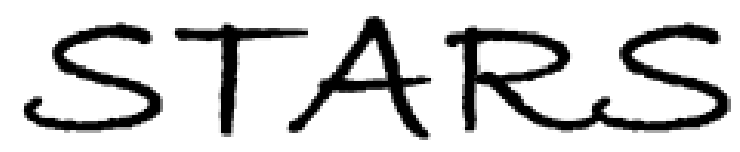

University of Central Florida

STARS

$1-1-2008$

\title{
Applicability of hybrid density functional theory methods to calculation of molecular hyperpolarizability
}

Kyrill Yu. Suponitsky

Sergio Tafur

University of Central Florida

Artëm E. Masunov

University of Central Florida

Find similar works at: https://stars.library.ucf.edu/facultybib2000

University of Central Florida Libraries http://library.ucf.edu

This Article is brought to you for free and open access by the Faculty Bibliography at STARS. It has been accepted for inclusion in Faculty Bibliography 2000 s by an authorized administrator of STARS. For more information, please contact STARS@ucf.edu.

\section{Recommended Citation}

Suponitsky, Kyrill Yu.; Tafur, Sergio; and Masunov, Artëm E., "Applicability of hybrid density functional theory methods to calculation of molecular hyperpolarizability" (2008). Faculty Bibliography 2000s. 1037. https://stars.library.ucf.edu/facultybib2000/1037

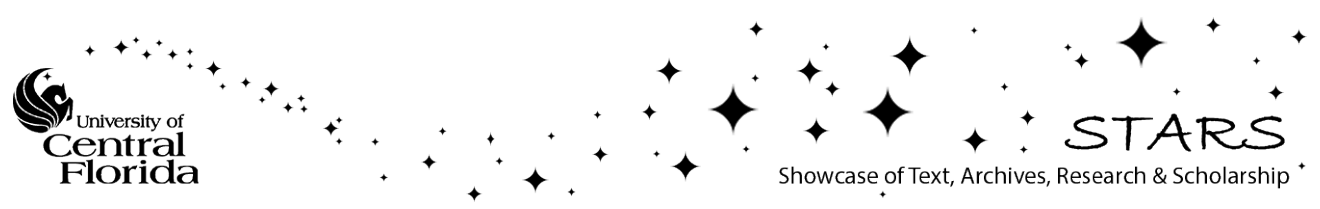




\section{Applicability of hybrid density functional theory methods to calculation of molecular hyperpolarizability}

Cite as: J. Chem. Phys. 129, 044109 (2008); https://doi.org/10.1063/1.2936121

Submitted: 25 February 2008 . Accepted: 01 May 2008 . Published Online: 30 July 2008

Kyrill Yu. Suponitsky, Sergio Tafur, and Artëm E. Masunov

ARTICLES YOU MAY BE INTERESTED IN
Polarizability and second hyperpolarizability evaluation of long molecules by the density
functional theory with long-range correction
The Journal of Chemical Physics 126, 014107 (2007); https:// doi.org/10.1063/1.2428291
Density-functional thermochemistry. III. The role of exact exchange
The Journal of Chemical Physics 98, 5648 (1993); https://doi.org/10.1063/1.464913
Problems in the comparison of theoretical and experimental hyperpolarizabilities
The Journal of Chemical Physics 97, 7590 (1992); https://doi.org/10.1063/1.463479

ARTICLES YOU MAY BE INTERESTED IN
Polarizability and second hyperpolarizability evaluation of long molecules by the density
functional theory with long-range correction
The Journal of Chemical Physics 126, 014107 (2007); https:// doi.org/10.1063/1.2428291
Density-functional thermochemistry. III. The role of exact exchange
The Journal of Chemical Physics 98, 5648 (1993); https://doi.org/10.1063/1.464913
Problems in the comparison of theoretical and experimental hyperpolarizabilities
The Journal of Chemical Physics 97, 7590 (1992); https://doi.org/10.1063/1.463479

ARTICLES YOU MAY BE INTERESTED IN
Polarizability and second hyperpolarizability evaluation of long molecules by the density
functional theory with long-range correction
The Journal of Chemical Physics 126, 014107 (2007); https:// doi.org/10.1063/1.2428291
Density-functional thermochemistry. III. The role of exact exchange
The Journal of Chemical Physics 98, 5648 (1993); https://doi.org/10.1063/1.464913
Problems in the comparison of theoretical and experimental hyperpolarizabilities
The Journal of Chemical Physics 97, 7590 (1992); https://doi.org/10.1063/1.463479

ARTICLES YOU MAY BE INTERESTED IN
Polarizability and second hyperpolarizability evaluation of long molecules by the density
functional theory with long-range correction
The Journal of Chemical Physics 126, 014107 (2007); https:// doi.org/10.1063/1.2428291
Density-functional thermochemistry. III. The role of exact exchange
The Journal of Chemical Physics 98, 5648 (1993); https://doi.org/10.1063/1.464913
Problems in the comparison of theoretical and experimental hyperpolarizabilities
The Journal of Chemical Physics 97, 7590 (1992); https://doi.org/10.1063/1.463479

ARTICLES YOU MAY BE INTERESTED IN
Polarizability and second hyperpolarizability evaluation of long molecules by the density
functional theory with long-range correction
The Journal of Chemical Physics 126, 014107 (2007); https://doi.org/10.1063/1.2428291
Density-functional thermochemistry. III. The role of exact exchange
The Journal of Chemical Physics 98, 5648 (1993); https://doi.org/10.1063/1.464913
Problems in the comparison of theoretical and experimental hyperpolarizabilities
The Journal of Chemical Physics 97, 7590 (1992); https://doi.org/10.1063/1.463479

ARTICLES YOU MAY BE INTERESTED IN
Polarizability and second hyperpolarizability evaluation of long molecules by the density
functional theory with long-range correction
The Journal of Chemical Physics 126, 014107 (2007); https:// doi.org/10.1063/1.2428291
Density-functional thermochemistry. III. The role of exact exchange
The Journal of Chemical Physics 98, 5648 (1993); https://doi.org/10.1063/1.464913
Problems in the comparison of theoretical and experimental hyperpolarizabilities
The Journal of Chemical Physics 97, 7590 (1992); https://doi.org/10.1063/1.463479

ARTICLES YOU MAY BE INTERESTED IN
Polarizability and second hyperpolarizability evaluation of long molecules by the density
functional theory with long-range correction
The Journal of Chemical Physics 126, 014107 (2007); https:// doi.org/10.1063/1.2428291
Density-functional thermochemistry. III. The role of exact exchange
The Journal of Chemical Physics 98, 5648 (1993); https://doi.org/10.1063/1.464913
Problems in the comparison of theoretical and experimental hyperpolarizabilities
The Journal of Chemical Physics 97, 7590 (1992); https://doi.org/10.1063/1.463479

ARTICLES YOU MAY BE INTERESTED IN
Polarizability and second hyperpolarizability evaluation of long molecules by the density
functional theory with long-range correction
The Journal of Chemical Physics 126, 014107 (2007); https:// doi.org/10.1063/1.2428291
Density-functional thermochemistry. III. The role of exact exchange
The Journal of Chemical Physics 98, 5648 (1993); https://doi.org/10.1063/1.464913
Problems in the comparison of theoretical and experimental hyperpolarizabilities
The Journal of Chemical Physics $\mathbf{9 7 , 7 5 9 0 ~ ( 1 9 9 2 ) ; ~ h t t p s : / / d o i . o r g / 1 0 . 1 0 6 3 / 1 . 4 6 3 4 7 9 ~}$

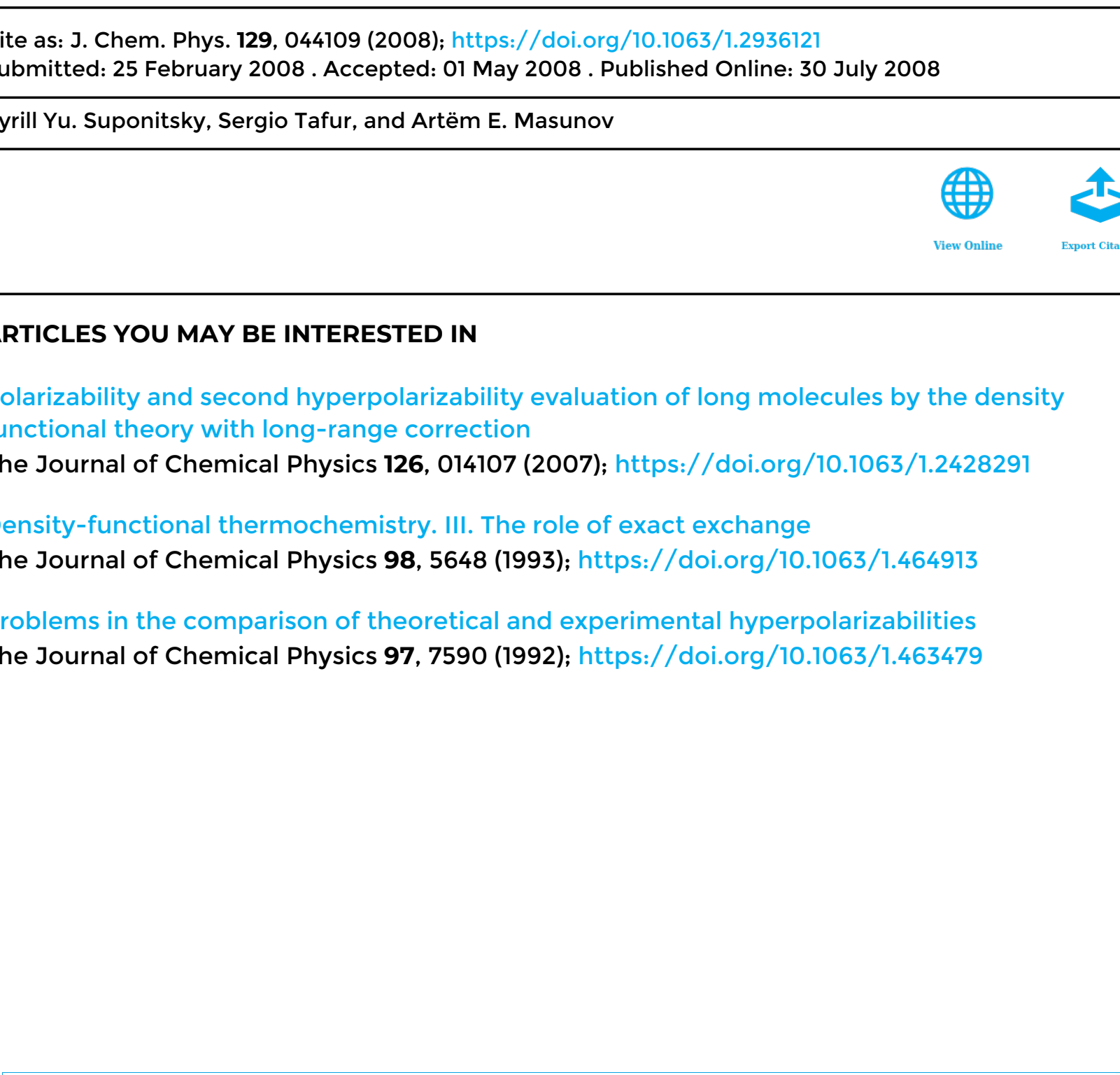

Lock-in Amplifiers up to $600 \mathrm{MHz}$

starting at

$\$ 6,210$

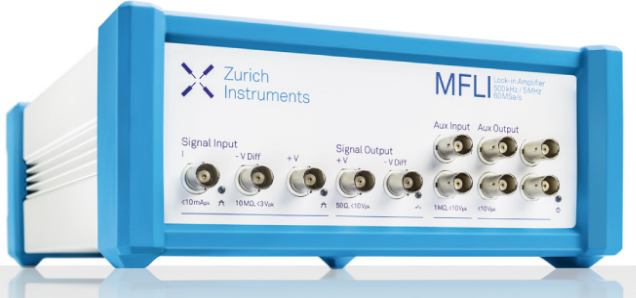

J. Chem. Phys. 129, 044109 (2008); https://doi.org/10.1063/1.2936121

(c) 2008 American Institute of Physics. 


\title{
Applicability of hybrid density functional theory methods to calculation of molecular hyperpolarizability
}

\author{
Kyrill Yu. Suponitsky, ${ }^{1, a)}$ Sergio Tafur, ${ }^{2}$ and Artëm E. Masunov ${ }^{3, b)}$ \\ ${ }^{1}$ Nanoscience Technology Center, University of Central Florida, Orlando, Florida 32826, USA and A.N. \\ Nesmeyanov Institute of Organoelement Compounds, Russian Academy of Sciences, 28, Vavilov \\ Street, B-334, Moscow 117813, Russia \\ ${ }^{2}$ Nanoscience Technology Center and Department of Physics, University of Central Florida, Orlando, \\ Florida 32826, USA \\ ${ }^{3}$ Nanoscience Technology Center, Department of Chemistry, and Department of Physics, University of \\ Central Florida, Orlando, Florida 32826, USA
}

(Received 25 February 2008; accepted 1 May 2008; published online 30 July 2008)

\begin{abstract}
The donor/acceptor (D/A) substituted $\pi$-conjugated organic molecules possess extremely fast nonlinear optical (NLO) response time that is purely electronic in origin. This makes them promising candidates for optoelectronic applications. In the present study, we utilized four hybrid density functionals (B3LYP, B97-2, PBE0, BMK), Hartree-Fock, and second order Møller-Plesset correlation energy correction, truncated at second-order (MP2) methods with different basis sets to estimate molecular first hyperpolarizability $(\beta)$ of D/A-substituted benzenes and stilbenes (D $=\mathrm{OMe}, \mathrm{OH}, \mathrm{NMe}_{2}, \mathrm{NH}_{2} ; \mathrm{A}=\mathrm{NO}_{2}, \mathrm{CN}$ ). The results of density functional theory (DFT) calculations are compared to those of MP2 method and to the experimental data. We addressed the following questions: (1) the accurate techniques to compare calculated results to each other and to experiment, (2) the choice of the basis set, (3) the effect of molecular planarity, and (4) the choice of the method. Comparison of the absolute values of hyperpolarizabilities obtained computationally and experimentally is complicated by the ambiguities in conventions and reference values used by different experimental groups. A much more tangible way is to compare the ratios of $\beta$ 's for two (or more) given molecules of interest that were calculated at the same level of theory and measured at the same laboratory using the same conventions and reference values. Coincidentally, it is the relative hyperpolarizabilities rather than absolute ones that are of importance in the rational molecular design of effective NLO materials. This design includes prediction of the most promising candidates from particular homologous series, which are to be synthesized and used for further investigation. In order to accomplish this goal, semiquantitative level of accuracy is usually sufficient. Augmentation of the basis set with polarization and diffuse functions changes $\beta$ by $20 \%$; however, further extension of the basis set does not have significant effect. Thus, we recommend $6-31+\mathrm{G}^{*}$ basis set. We also show that the use of planar geometry constraints for the molecules, which can somewhat deviate from planarity in the gas phase, leads to sufficient accuracy (with an error less than 10\%) of predicted values. For all the molecules studied, MP2 values are in better agreement with experiment, while DFT hybrid methods overestimate $\beta$ values. BMK functional gives the best agreement with experiment, with systematic overestimation close to the factor of 1.4. We propose to use the scaled BMK results for prediction of molecular hyperpolarizability at semiquantitative level of accuracy. (C) 2008 American Institute of Physics.
\end{abstract}

[DOI: $10.1063 / 1.2936121]$

\section{INTRODUCTION}

Over the past two decades, organic materials are rapidly gaining practical interest for nonlinear optical (NLO) applications. ${ }^{1}$ The donor and/or acceptor substituted $\pi$-conjugated organic molecules possess extremely fast NLO response time that is purely electronic in origin. This makes them promising candidates for optoelectronic applications. Design of the molecules with high molecular first hyperpolarizabilities $(\beta)$ and appropriate optical transparency is an

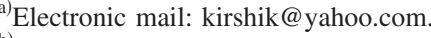

b) Author to whom correspondence should be addressed. Electronic mail: amasunov@mail.ucf.edu.
}

important step in the quest for effective NLO materials. The use of quantum chemical methods for prediction of the molecular NLO properties is expected to provide a guidance and accelerate subsequent experimental studies.

The reliable estimation of NLO properties requires theory levels that include electron correlation and large basis sets with polarization as well as diffuse functions., ${ }^{2,3}$ It was shown that second-order Møller-Plesset correlation energy correction, truncated at second-order (MP2) and coupled cluster methods can reproduce molecular hyperpolarizabilities with high accuracy. ${ }^{4,5}$ However, their application to molecules of practical interest built up of more than dozen of atoms can hardly be considered a routine task. Taking into 
account the feasibility of the density functional theory (DFT) to large molecules, ${ }^{6}$ this method presents a promising tool for this purpose.

The reliability of DFT for estimation of the molecular NLO properties was criticized. ${ }^{7-9}$ Inadequacies of pure DFT functionals [both the local density approximation and the generalized gradient approximation (GGA)] were identified as wrong asymptotic behavior of the functional and selfinteraction error. Since these sources of error are absent in Hartree-Fock (HF) method, the fraction of HF exchange proportionally reduces but does not eliminate the inaccuracies of pure DFT. Several approaches were proposed to improve the situation. Asymptotically corrected LB94 functional was shown to significantly overestimate the molecular hyperpolarizability of highly polarizable systems such as $\mathrm{NH}_{2} / \mathrm{NO}_{2}$-terminated long-chain polyenes. In another approach, gradient-regulated asymptotic connection procedure was applied to Becke and Perdew GGA potential termed BP-GRAC (shape-corrected) ${ }^{10}$ method. Its success in the estimation of hyperpolarizabilities of small molecules was demonstrated. ${ }^{9}$ However, in the recent study ${ }^{11}$ of solvent dependence of para-nitroaniline ( $p$-NA), BP-GRAC method was shown to twice overestimate the value of first hyperpolarizability. At the same time, according to comparison given in Ref. 5, conventional DFT methods B3LYP and B971 give similar quality results for small molecules.

Based on comparison with MP2 results, both pure DFT and hybrid GGA DFT methods overestimate $\beta,{ }^{8}$ but hybrid GGA performs better. In the study of 4-amino-4'-nitrostilbene (ANS), the ratio of $\beta$ for ANS/pNA was reported to be 5.2 for MP2 and 11.8 for B3LYP, while for the $\mathrm{NH}_{2}-(\mathrm{CH}=\mathrm{CH})_{12}-\mathrm{NO}_{2}$ molecule B3LYP hyperpolarizability is greater than that of MP2 method by the factor of 3.2. Based on these data, Champagne et al. ${ }^{8}$ concluded that DFT fails to correctly reproduce molecular hyperpolarizability; however, no experimental data were used for its verification. One may also doubt if MP2/6-31G level of theory is accurate enough to serve for the benchmarking purposes. The reported experimental values of the $\beta_{\text {(D/A-substituted stilbene) }} / \beta_{(\mathrm{D} / \mathrm{A} \text {-substituted benzene) }}$ ratio vary between 5 and $10,{ }^{5,12}$ depending on D/A strength and solution used in electric field-induced second harmonic generation (EFISH) measurements. Based on these experimental ratios, MP2/6$31 \mathrm{G}$ level of theory somewhat underestimates hyperpolarizabilities of long-chain $\pi$-conjugated systems. While D/Asubstituted polyenes often have low thermal stability and are not optically transparent, the molecules of practical interest usually contain combination of aromatic and ethylene fragments. The representative series of such molecules was recently considered by Robinson et al. ${ }^{13}$ The authors came to conclusion that molecules containing up to eight conjugated double bonds in the $\pi$-system are in the "safe domain" to be systematically studied by conventional DFT methods at least at a qualitative level. This should be sufficient for the practical purposes, as the systematic design of the improved materials for NLO applications primarily requires the knowledge of structure-activity relations in hyperpolarizabilities and the trends rather then absolute values to provide a guidance to the experimentalists.
Another important question is the choice of a basis set. An accurate description of hyperpolarizabilities may require basis sets of high quality. One approach is to use multipurpose basis sets obtained from variational principle of minimal total energy and systematically converging to the infinite basis limit. The examples are the augmented correlationconsistent basis sets aug-cc-pVXZ of Dunning ${ }^{14}$ or polarization-consistent sets $\mathrm{pc}-\mathrm{X}$ of Jensen. ${ }^{15}$ Another approach is to keep a medium-sized basis set and optimize the exponents of the basis functions to accurately reproduce specific molecular property of choice. An example of such a custom-designed basis sets was given by Chong et al. ${ }^{16}$ They used perturbation theory approach to derive field-induced polarization (FIP) functions for hydrogenic atom ${ }^{17}$ and extrapolated the STO exponents to the atoms from $\mathrm{H}$ to $\mathrm{Kr}$. The first-order functions (FIP1) were designed for calculations of $\alpha$ and $\beta$, while second-order functions (FIP2) are necessary for calculations of the second hyperpolarizability $\gamma$. For several small molecules, where the sum of atomic polarizabilities presents a good estimate for molecular polarizability, the results were found encouraging. However, for alkali diatomics, where charge redistribution between the atoms dominates polarizability and hyperpolarizability, the addition of FIP yields poor results, deteriorating with the size of the basis set. Similarly, static second hyperpolarizability of benzene and borazine was found ${ }^{18}$ to be $30 \%$ smaller with FIP functions than respective predictions obtained in a large even-tempered basis set.

A more successful approach to the design of basis sets for description of dipole moments and polarizabilities was suggested by Sadlej (Pol basis). ${ }^{19}$ In this basis set, generation of polarization functions follows from the well-defined physical model for the dependence of the electron density distribution on the strength of the external electric field. ${ }^{20}$ Nonlinear polarizabilities require an extension of the Pol basis set: Pol++ by Bartlett et al. ${ }^{21,22}$ and HyPol by Sadlej et $a .^{23}$ The theoretical study on urea and thiourea ${ }^{24}$ demonstrates that this extension is essential to obtain the accurate second hyperpolarizability values. However, such a large basis set can become impractical to handle big-size molecules. Recently, the reduction in Pol basis to ZmPol was suggested by Sadlej et al. ${ }^{25}$ The Zm3Pol basis was tested versus augcc-pVTZ by Leszczynski et $a{ }^{3}{ }^{3}$ and provided the reasonable agreement with larger basis set results for NLO properties of urea, fluoroacetylene, and diformamide.

In their time-dependent DFT (TD-DFT) study of the third-order polarizabilities, Masunov and Tretiak $^{26}$ have shown that split-valence basis set 6-31G is sufficient, and the results do not significantly change when standard polarization $(*)$ and diffuse functions $(+)$ are added. This is in agreement with some of the data from Ref. 2. One may notice that for multiconfigurational methods (as opposed to $\mathrm{HF}$ and DFT) the use of 6-31G basis may lead, in some cases, to completely unrealistic results while $6-31+G^{*}$ is sufficient for qualitative predictions. ${ }^{2}$

The main goal of the present study is to analyze the predictive capability of hybrid DFT methods based on comparison with the results of experimental measurements. An important aspect is internal consistency in the benchmark set 
<smiles>[NH][14c]1[14cH][14cH][14c]([N+](=O)[O-])[14cH][14cH]1</smiles>
$p \mathrm{NA}$<smiles>COc1ccc([N+](=O)[O-])cc1</smiles><smiles>O=[N+]([O-])c1ccc(O)cc1</smiles><smiles>CN(C)c1ccc(C#N)cc1</smiles><smiles>CN(C)c1ccc([N+](=O)[O-])cc1</smiles>

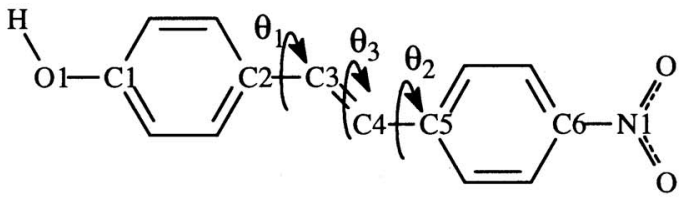

HONS

MONB

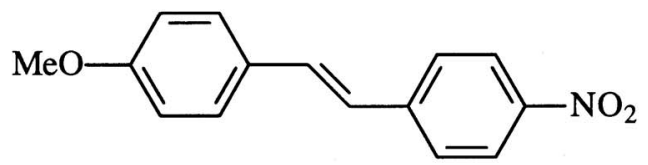

\section{MONS}

HONB

DACB

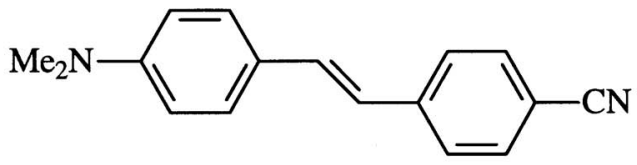

DANB

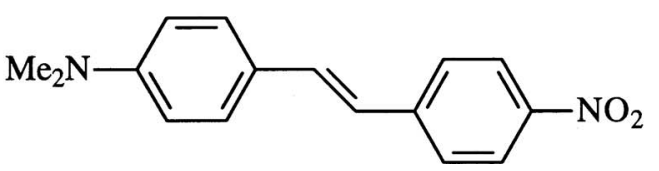

DANS

SCHEME 1.

of the experimental data. Comparison of the calculated hyperpolarizabilities to experimental values could be problematic due to the use of different conventions in the definitions of $\beta$ and standards by experimentalists. This problem was specially addressed recently. ${ }^{27-29}$ In review, ${ }^{29}$ it was not recommended to compare results obtained by different authors. For $p$-NA molecule, most of experimental NLO data were critically analyzed and tabulated in unified scale by Reis. ${ }^{28}$ At the present time, para-nitroaniline is the only prototype of a push-pull molecule with D/A substituents connected by the $\pi$-conjugated bridge that has a reliably determined value of molecular hyperpolarizability in the gas phase. This allows for a direct comparison with the absolute $\beta$ values calculated for an isolated molecule. Unfortunately, all the other accurate gas-phase measurements were carried out for small molecules such as water, acetonitrile, etc., and may be of interest only for the benchmarking of higher theory levels.

The NLO properties of $p$-NA have been the subject of theoretical study in several publications. Salek et al. ${ }^{30}$ have found that the B3LYP value of $\beta$ of $p$-NA is in good agreement with that of the CCSD method. Yang et al. ${ }^{31}$ have recently calculated hyperpolarizability of $p$-NA using the TDDFT/sum over state (SOS) formalism. They considered several functionals, including pure DFT as well as hybrid GGA. The hybrid functionals were shown to perform better, in agreement with the other literature data. ${ }^{8}$ Yang et al. also concluded that $\beta$ of $p$-NA is nearly insensitive to the choice of a basis set, in contrast to the earlier findings, ${ }^{2,3}$ which showed that augmentation of the basis set with both polarization and diffuse functions is necessary to obtain reliable values of NLO properties. At the same time, another study by Soscun et ll $^{32}$ found that hyperpolarizability of $p$-NA do not significantly vary in the series of basis sets with both polarization and diffuse functions.

Several computational works ${ }^{33-35}$ investigated the sol- vent dependence of molecular geometry and $\beta$ for $p$-NA, using polarizable continuum model (PCM). The geometry characteristics of $p$-NA, most sensitive to solvent effect, were found to be $\mathrm{C}-\mathrm{N}$ bond lengths. ${ }^{33,35}$ For instance, transition from the gas phase to the acetone solution changes the $\mathrm{C}-\mathrm{N}$ bonds by $0.01-0.02 \AA{ }^{35}$ These changes are accompanied by a strong enhancement of hyperpolarizability, which is in agreement with the earlier experimental observations (see, for instance, Ref. 28). At the same time, more recent measurements found the peak resonant hyperpolarizability and the two-photon absorption cross section of $N, N$-dipropyl substituted $p$-NA to be nearly solvent independent. $^{36}$

In the present work, we report the systematic study of the basis set and DFT functional dependence on the static first hyperpolarizability. The reliability of different DFT methods for prediction of hyperpolarizability enhancement with the increasing length of a conjugated $\pi$-system is also studied. To compensate the ambiguity in experimental data, we compare ratios of calculated hyperpolarizabilities for two molecules with experimental ratios (measured in the same laboratory) rather than absolute values. Among the available experimental data, we have selected several pairs of D- $\pi$-A molecules: One with the benzene $\pi$-system and the other with the stilbene $\pi$-system. Hyperpolarizabilities for those molecules (Scheme 1) were calculated taking into account both solvent and frequency dependence for a rigorous comparison with the experiment. Frequency-dependent hyperpolarizabilities are estimated with both coupled perturbed HF (CPHF) (Ref. 37) and SOS (Ref. 38) methods and their numerical results are compared.

To study the basis set dependence, we have chosen two benchmark molecules. The first molecule is $p$-NA, which is a representative of the medium-sized $\pi$-systems. Both experimental $^{28,39}$ and theoretical hyperpolarizabilities, as 
well as the X-ray structure ${ }^{40}$ are available for $p$-NA. Our second benchmark molecule with a longer $\pi$-system is 4-hydroxy-4' -nitrostilbene (HONS). Its NLO properties are also well characterized ${ }^{41}$ and its crystal structure is also determined by the X-ray method. ${ }^{42}$

\section{THEORY}

The dipole moment vector $\mu_{i}(i=x, y, z)$ can be defined as a negative derivative of energy with respect to the external electric field in the limit of a vanishing field strength,

$$
\mu_{i}=-\left.\frac{\partial W(E)}{\partial E_{i}}\right|_{E=0} .
$$

When a molecule is placed in an electric field of the strength $E_{i}$, its dipole moment can be expressed as the Taylor expansion series in the order of the field strength,

$$
\mu_{i}=\mu_{i}^{0}+\alpha_{i j} E_{j}+\beta_{i j k} E_{j} E_{k}+\gamma_{i j k l} E_{j} E_{k} E_{l}+\cdots,
$$

where $\mu_{i}^{0}$ is the dipole moment of the unperturbed molecule, $\alpha_{i j}$ is the linear polarizability, and $\beta_{i j k}$ and $\gamma_{i j k l}$ are the first and second hyperpolarizabilities, respectively. Therefore, in computational chemistry tools, (hyper)polarizabilities are estimated as derivatives of the dipole moment with respect to the applied field. This can be done numerically [finite field (FF) approach] or analytically with CPHF method. Hyperpolarizability is also the third derivative of energy $(W)$ with respect to the applied field,

$$
\beta_{i j k}=-\left.\frac{\partial^{3} W(E)}{\partial E_{i} \partial E_{j} \partial E_{k}}\right|_{E=0} .
$$

Only certain components of this third-order tensor are available from experiment. Specifically, the measurements of EFISH yields the product of the dipole moment and vectorial part of $\beta$ ( $\left.\beta_{\text {vect }}\right)$,

$$
\beta_{\mathrm{vect}}=\left(\beta_{x}^{2}+\beta_{y}^{2}+\beta_{z}^{2}\right)^{1 / 2}
$$

where

$$
\beta_{j}=\frac{1}{3} \sum_{i=1}^{3}\left(\beta_{j i i}+\beta_{i j i}+\beta_{i i j}\right), \quad i, j=x, y, z .
$$

If a dipole moment value is available from independent measurements, EFISH results are reported as $\beta_{\text {vect }}$ in assumption of collinearity between $\mu$ and $\beta_{\text {vect }}$.

An external electric field can be either static $(\omega=0)$ or dynamic, characterized by the frequency $\omega$ in the optical range. The frequency dependence of hyperpolarizability is also known as dispersion. EFISH experiments are typically performed at $\omega=0.65 \mathrm{eV}(\lambda=1907 \mathrm{~nm})$ or $1.17 \mathrm{eV} \quad(\lambda$ $=1064 \mathrm{~nm}$ ). Within the GAUSSIAN 2003 program, ${ }^{43}$ employed in the present study, analytic dynamic hyperpolarizability is implemented only at the HF level of theory. For the DFT, MP2, and CCSD methods, which do account for the electron correlation, one can use the multiplicative HF correction to a static correlated property. ${ }^{21,44}$ In this approximation, dynamic hyperpolarizability can be expressed as

$$
\begin{aligned}
& \beta_{\mathrm{corr}}(2 \omega, \omega, \omega)=\beta_{\mathrm{corr}}(0) \frac{\beta_{\mathrm{HF}}(2 \omega, \omega, \omega)}{\beta_{\mathrm{HF}}(0)}=\beta_{\mathrm{corr}}(0) \times \Delta, \\
& \Delta=\frac{\beta_{\mathrm{HF}}(2 \omega, \omega, \omega)}{\beta_{\mathrm{HF}}(0)},
\end{aligned}
$$

where $\beta_{\text {corr }}(0)$ and $\beta_{\text {corr }}(2 \omega, \omega, \omega)$ are static and dynamic hyperpolarizabilities at the correlated level of theory, and $\beta_{\mathrm{HF}}(0)$ and $\beta_{\mathrm{HF}}(2 \omega, \omega, \omega)$ are the same properties calculated at HF level; $\Delta$ is the multiplicative correction factor.

Another method to calculate hyperpolarizability is given by the SOS approach. ${ }^{38}$ Its advantage over the FF method is that the frequency dependence is explicitly included in the expression for the components of the first hyperpolarizability tensor, which can be written as

$$
\begin{aligned}
\beta(\omega)_{i j k}= & -\frac{1}{\hbar^{2}} \sum_{n} \sum_{n^{\prime}}\left[\left(r_{g n^{\prime}}^{j} r_{n^{\prime} n}^{i} r_{g n}^{k}+r_{g n^{\prime}}^{k} r_{n^{\prime} n}^{i} r_{g n}^{j}\right)\left(\frac{1}{\left(\omega_{n^{\prime} g}-\omega\right)\left(\omega_{n g}+\omega\right)}+\frac{1}{\left(\omega_{n^{\prime} g}+\omega\right)\left(\omega_{n g}-\omega\right)}\right)\right. \\
& +\left(r_{g n^{\prime}}^{i} r_{n^{\prime} n}^{j} r_{g n}^{k}+r_{g n^{\prime}}^{i} r_{n^{\prime} n}^{k} r_{g n}^{j}\right)\left(\frac{1}{\left(\omega_{n^{\prime} g}+2 \omega\right)\left(\omega_{n g}+\omega\right)}+\frac{1}{\left(\omega_{n^{\prime} g}-2 \omega\right)\left(\omega_{n g}-\omega\right)}\right) \\
& \left.+\left(r_{g n^{\prime}}^{j} r_{n^{\prime} n}^{k} r_{g n}^{i}+r_{g n^{\prime}}^{k} r_{n^{\prime} n}^{j} r_{g n}^{i}\right)\left(\frac{1}{\left(\omega_{n^{\prime} g}-\omega\right)\left(\omega_{n g}-2 \omega\right)}+\frac{1}{\left(\omega_{n^{\prime} g}+\omega\right)\left(\omega_{n g}+2 \omega\right)}\right)\right],
\end{aligned}
$$

where the transition dipole moment operator $r$ and the frequencies $\omega$ with subscripts correspond to the transitions between the ground $(g)$ and excited $\left(n, n^{\prime}\right)$ states. Although the expectation values of state-to-state and permanent excited state dipole moment operators can be readily evaluated in configuration interaction (CI) singles method, these expectation values are not available in full linear response approach, such as TDHF and TDDFT. In order to estimate these values, 
TABLE I. Main geometry characteristics ( $\AA$, deg), hyperpolarizabilities ( $\beta$, a.u.), dipole moment $(\mu, \mathrm{D})$, and charge distribution $(q, e)$ dependence on the variation of the basis set for $p$-NA in $C_{s}$ geometry (the values of $\beta$ are also presented for $\mathrm{C}_{2 v}$ and $\mathrm{X}$-ray geometries) calculated with B97-2 functional.

\begin{tabular}{lccccc}
\hline \hline $\begin{array}{l}\text { Geometry characteristic } \\
\text { or property }\end{array}$ & $6-31 \mathrm{G}$ & $6-31 \mathrm{G}(d)$ & $6-31 \mathrm{G}(2 d)$ & $6-31+\mathrm{G}$ & $6-31+\mathrm{G}(d)$ \\
\hline $\mathrm{N} 1-\mathrm{C} 1$ & 1.368 & 1.375 & 1.380 & 1.370 & 1.376 \\
$\mathrm{C} 1-\mathrm{C} 2$ & 1.414 & 1.409 & 1.405 & 1.415 & 1.409 \\
$\mathrm{C} 2-\mathrm{C} 3$ & 1.384 & 1.383 & 1.381 & 1.385 & 1.384 \\
$\mathrm{C} 3-\mathrm{C} 4$ & 1.399 & 1.394 & 1.391 & 1.400 & 1.396 \\
$\mathrm{C} 4-\mathrm{N} 2$ & 1.442 & 1.452 & 1.454 & 1.441 & 1.453 \\
$\angle \mathrm{NH}_{2}, \mathrm{Ph}$ & 0.0 & 31.1 & 38.2 & 0.0 & 30.1 \\
$\beta_{\mathrm{Cs}}$ & 1683 & 1257 & 1158 & 2116 & 1596 \\
$\beta_{\mathrm{C} 2 v}$ & 1683 & 1299 & 1226 & 2116 & 1655 \\
$\beta_{x \text { ray }}$ & 1558 & 1326 & 1280 & 1940 & 1675 \\
$\mu$ & 8.3 & 7.1 & 6.6 & 8.6 & 7.4 \\
$q_{\mathrm{NH} 2}$ & 0.029 & 0.003 & -0.004 & 0.028 & 0.005 \\
$q_{\mathrm{C} 1}$ & 0.196 & 0.198 & 0.184 & 0.189 & 0.189 \\
$q_{\mathrm{C} 2(\mathrm{H})}$ & -0.045 & -0.045 & -0.040 & -0.040 & -0.041 \\
$q_{\mathrm{C} 3(\mathrm{H})}$ & 0.086 & 0.081 & 0.083 & 0.091 & 0.085 \\
$q_{\mathrm{C} 4}$ & 0.021 & 0.011 & 0.004 & 0.031 & 0.016 \\
$q_{\mathrm{NO} 2}$ & -0.329 & -0.283 & -0.270 & -0.350 & -0.299 \\
\hline \hline
\end{tabular}

one must apply quadratic response approach, ${ }^{45}$ or resort to the a posteriori Tamm-Dancoff approximation, which was recently introduced, implemented, and validated against Symmetry Adapted Cluster/Configuration Interaction (SACCI) benchmarking results. ${ }^{46}$

\section{COMPUTATIONAL DETAILS}

The GAUSSIAN 2003 program $^{43}$ was used for all calculations. Several exchange-correlation functionals such as B97-2, ${ }^{47}$ B3LYP, ${ }^{48}$ PBE0 (Ref. 49) (hybrid GGA), and BMK (Ref. 50) (hybrid meta-GGA) in DFT, as well as HF and correlated methods MP2 and CCSD were chosen for this study. As hybrid functionals are more suitable for calculations of NLO properties, we did not consider pure exchangecorrelation functionals, which do not include a fraction of HF exchange. Detailed study of the basis set effects was carried out using the B97-2 functional. This method was chosen as an improvement over B97-1, recommended for estimation of second-order optical properties and being superior to the widely used B3LYP functional. ${ }^{5,51}$ The FF method was used to calculate hyperpolarizabilities with DFT, MP2, and CCSD methods (Freq=Raman keyword). At the HF level, the CPHF method with analytical derivatives was used (Polar=DCSHG keyword). The SOS method was utilized for calculation of hyperpolarizabilities (static and dynamic) at TDHF and TDDFT levels using the in-house FORTRAN code NLO. ${ }^{52}$ State-to-state and permanent excited state dipoles that are input to NLO, were calculated in the a posteriori Tamm-Dancoff approximation, implemented in the locally modified version of GAUSSIAN 2003 . $^{46}$

Availability of reliable experimental values of the gasphase hyperpolarizability of $p$-NA allows a direct comparison with our calculated data. For the HONS, we used the ratio $\beta_{(\mathrm{D} / \mathrm{A} \text {-substituted stilbene })} / \beta_{\text {(D/A-substituted benzene) }}$, where both values were taken from the same study (in the same solvent and at the same wavelength of the incident beam) so that uncertainties associated with a particular convention, a benchmark choice, and the experimental setup cancel. For other pairs of the molecules on Scheme 1, static hyperpolarizabilities in solution were corrected for dispersion using Eq. (4), while solvation effects were accounted for using the polarizable continuum model $(\mathrm{SCRF}=\mathrm{IEFPCM}$ with the option NoAddSph).

\section{RESULTS AND DISCUSSION}

\section{A. $p$-NA}

The properties of the $p$-NA calculated with B97-2 functional are presented in Table I (the structure and properties predicted with other methods, basis sets, and symmetry are deposited with the Electronic Physics Auxiliary Publication Service EPAPS of the American Institute of Physics ${ }^{53}$ ). In order to separate the effect of molecular geometry from one of the theory level, we carried out calculations with both optimized and fixed geometry. The X-ray geometry was used to investigate the dependence of hyperpolarizability on the theory level. In a crystal, $p$-NA adopts nearly planar geometry with the planar amino group slightly rotated out of the plane of the benzene ring. On the contrary, the optimized structures of $p$-NA with all basis sets (except for those without polarization functions) predict the amino group to be pyramidalized. This is in agreement with the recent studies by Reis et al. ${ }^{54}$ and Rashid, ${ }^{55}$ who have concluded from indirect evidence that the gas-phase structure of the $p$-NA is not planar (characterized by the $C_{s}$ symmetry). Unfortunately, there are no experimental data that can quantify the nonplanarity of $p$-NA in the gas phase. Planarization of the amino group in the absence of polarization functions was reported earlier ${ }^{56}$ and polarization functions are required at least for the nitrogen atom. On the other hand, a further extension of the basis set to G3 at the MP2 theory level led to planarization of the amino group in an urea molecule after 
including the zero-point vibrational correction, as reported by Masunov and Dannenberg. ${ }^{57}$ To study the effect of pyramidalization on the geometry and hyperpolarizability we also carried out calculations for the optimized planar $\left(C_{2 v}\right)$ structure of $p$-NA.

If one considers the effects of the basis set on hyperpolarizability, the most pronounced changes in $\beta$ occur when the 6-31G basis set is augmented with polarization or diffuse functions. Addition of the first set of $d$-functions on $\mathrm{C}, \mathrm{N}, \mathrm{O}$ atoms causes a decrease in hyperpolarizability by $18 \%$, which is related to sizable changes in geometry (pyramidalization of the amino group, elongation of the $\mathrm{C}-\mathrm{N}$ bonds, shortening of the $\mathrm{C}-\mathrm{C}$ bond) and to a decrease in conjugation. The second set of $d$-functions leads to a decrease in $\beta$ by $4 \%$ only and further decrease in conjugation. At the same time, even larger effect is observed when diffuse functions are added to the $6-31 \mathrm{G}$ basis set; the increase in $\beta$ is $25 \%$ while only minor changes in geometry are observed. Below, we discuss these findings in more detail using the atomic charges and the dipole moments that are also reported in Table I.

The addition of the first set of $d$-functions leads to a decrease in the negative charge of the acceptor nitro group (by $0.05 e$ ) and a decrease in the positive charge on the donor amino group (by $0.02 e$ ). Also, a decrease in the positive charge of the $\mathrm{C} 4$ atom (by $0.01 e$ ) is observed. This leads to the $17 \%$ decrease in the dipole moment and corresponding decrease in the electron delocalization. The addition of the second set of $d$-functions causes significantly smaller changes in the atomic charges $(\sim 0.01 e)$ and the $8 \%$ decrease in the dipole moment. These changes can be interpreted as a balance shift in description of the interatomic and intraatomic polarization while charges reflect interatomic effects and $\beta$ reflects both. With the small 6-31G basis set, the basis functions centered on the neighboring atoms are used to essentially compensate for the deficiency of the basis set. This leads to the artifactual delocalization. The addition of the second set of $d$-functions mostly improves intra-atomic polarization, and has a smaller effect on the delocalization.

The situation with the diffuse functions is more complicated. Augmentation of the basis set with diffuse functions leads to the $0.02 e$ increase in the negative charge of the acceptor group and the $0.01 e$ increase in the positive charge of the $\mathrm{C} 4$ atom. Changes in the other charges are less than $0.01 e$ and dipole moment increase is marginal $(0.3 \mathrm{D})$. Thus, only a small increase in conjugation is observed, while a significant increase in the $\beta$ value is found. This can be interpreted as an intra-atomic charge redistribution that leads to the weakening of the electron-nucleus interaction. This weakening does not affect the integral atomic properties of the ground state while the effect on the properties related to an electron excitation is significant. The results obtained with the $6-31+\mathrm{G}^{*}$ basis display a nearly additive cancellation of the opposite effects originating from polarization and diffuse functions.

The addition of * and + functions to the basis set causes the most pronounced changes in the molecular geometry and hyperpolarizability. All the other modifications do not lead to significant changes in the predicted properties (Table I in
Ref. 53). The addition of polarization and diffuse functions on hydrogen atoms has a negligible effect on both geometry and hyperpolarizability. The minor effect is observed when a set of $f$-functions is added. Switch from the 6-31 family to 6-311 one causes a decrease in hyperpolarizability by $1.5 \%$. The augmentation of the basis with the second set of diffuse functions, or with Rydberg functions suggested by Kaufmann, ${ }^{58}$ results in a marginal decrease in hyperpolarizability (less than $2 \%$ ).

From Table I in Ref. 53, one can conclude that hyperpolarizability does not depend significantly on a choice of the geometry. Dependence of $\beta$ on the basis set has the same trends for $C_{s}, C_{2 v}$, and X-ray structures. All basis sets show a small increase $(\sim 2 \%-4 \%)$ in the series $C_{s}<C_{2 v}<\mathrm{X}$-ray (except for the basis sets without polarization functions). This may be explained by increased electron conjugation in $\mathrm{X}$-ray structure due to the effect of the crystal field, and decreased conjugation in the bent $C_{s}$ structure. The decreases in $\beta$ from $6-31 \mathrm{G}$ to $6-31 \mathrm{G}(\mathrm{d})$ are $25 \%, 23 \%, 15 \%$ for $C_{s}$, $C_{2 v}$, and X-ray geometries, respectively. This change is much smaller from 6-31G(d) to 6-31G(2d) and a further basis set expansion. This observation clearly shows that for series of molecules with similar structures or for the study of the basis set dependence for the same molecule, the choice of the planarity constraints is insignificant (smaller than the accuracy of theoretical predictions).

The values of $\beta$ estimated by other methods with the $6-31+\mathrm{G}^{*}$ basis set are 1571, 1745, 1557, 1725, 1430, and 726 a.u. for PBE0, B3LYP, BMK, MP2, CCSD, and HF methods, respectively. The dependence of $p$-NA geometry and properties on a basis set for those methods agree with trends found for the B97-2 functional (Tables II and III in Ref. 53). One can notice that the HF method significantly underestimates hyperpolarizability and produces the bond lengths distribution and dipole moments that are inconsistent with DFT and MP2 data. For instance, HF/6-31+G(d) approximation leads to the shortening of $\mathrm{C}-\mathrm{N}\left(\mathrm{O}_{2}\right)$ and all aromatic $\mathrm{C}-\mathrm{C}$ bonds, and to the slight elongation of $\mathrm{C}-\mathrm{N}\left(\mathrm{H}_{2}\right)$ bonds (compare to B97-2 results). At the same time, the the HF dipole moment is only slightly lower than the one obtained at the B97-2/6-31+G(d) level. The advantage of the HF method, however, is that it allows an analytical evaluation of the frequency dependence of hyperpolarizability. ${ }^{21,44}$ For the basis sets with a single set of polarization and diffuse functions, a further extension of the basis set does not lead to any sizable changes in correction for the frequency dependence (Table III in Ref. 53). Thus, $\Delta=1.33$ can be used for $p$-NA.

The fact that for all the methods considered the most pronounced changes in $\beta$ are found when the basis set is augmented with the first set of polarization and diffuse functions for heavy atoms $(\mathrm{C}, \mathrm{N}, \mathrm{O}$ for $p$-NA) allows us to recommend the $6-31+\mathrm{G}(d)$ basis set for the estimation of hyperpolarizability while $6-31 \mathrm{G}(d)$ basis set seems to provide enough accuracy for estimation of the molecular geometry.

\section{B. 4-hydroxy-4'-nitrostilbene}

The experimental geometry of HONS is available from $\mathrm{X}$-ray study of its cocrystals with 4-cyano- and 


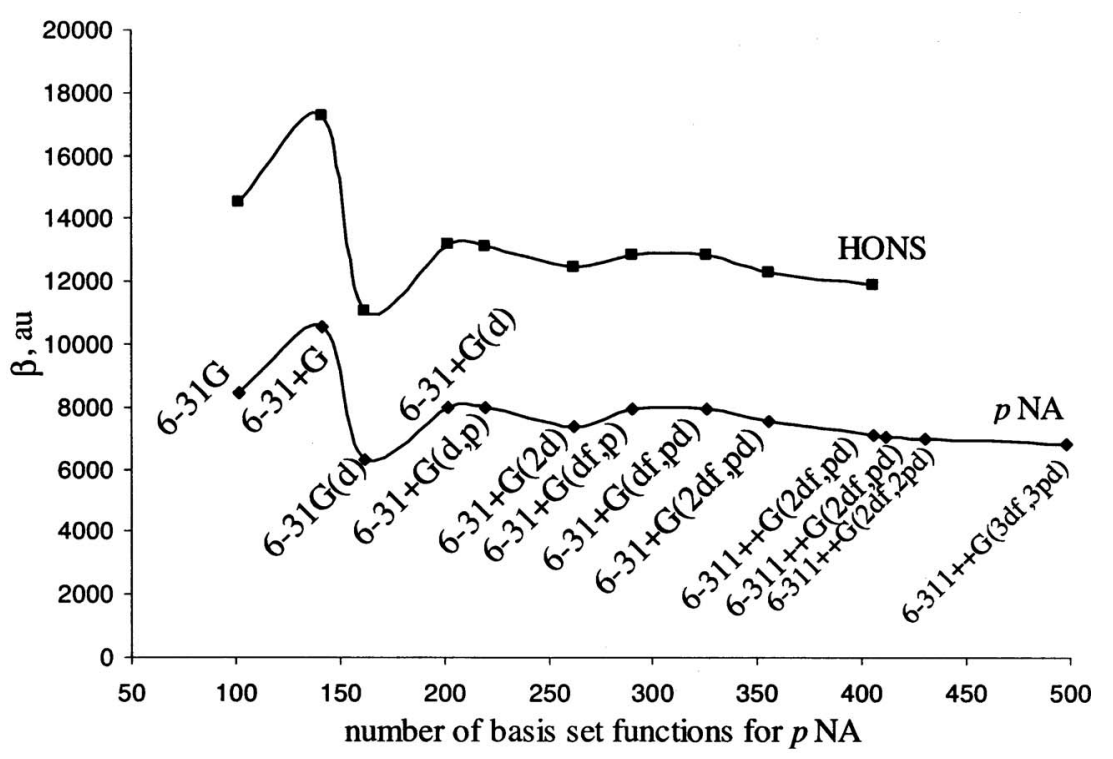

FIG. 1. Basis set dependence of $\beta(p$-NA) (multiplied by a factor of 5 for better visualization) and $\beta$ (HONS) for the B97-2 functional.

4-methyl-pyrindine-1-oxide. ${ }^{42}$ In both structures, the $\mathrm{OH}$ group is nearly coplanar to the adjacent benzene ring and is in trans orientation to the central $\mathrm{C} 3=\mathrm{C} 4$ double bond. This is in agreement with the findings based on fluorescent spectroscopy. ${ }^{59}$ Similar to the case of $p$-NA, there is no direct experimental data on the conformation of D/A-stilbenes in the gas phase. Based on fluorescence spectroscopic study, the planar $C_{2 h}$ structure was proposed for the unsubstituted stilbene. ${ }^{60}$ One should expect that substitution of stilbene with D/A groups results in enhanced conjugation and, therefore, planarization. However, fluorescence spectroscopic studies suggested nonplanar structure for 4-dimethylamino-4'-cyanostilbene (DACS), ${ }^{61}$ and planar structures for 4-methoxystilbene and 4-hydroxystilbene. ${ }^{59}$ Nearly planar conformation of HONS found in the X-ray study ${ }^{42}$ implies a strong conjugation. However, in both structures the lengths of $\mathrm{C} 2-\mathrm{C} 3[1.477(5), 1.508(5) \AA]$ and C4-C5 [1.469(4), 1.478(5) ^] bonds are too long, while the central double bond is extremely short $[1.319(5)$, $1.295(5) \AA]$. This is probably related to the disorder that is not mentioned in Ref. 42 and makes it impossible to compare the hyperpolarizabilities of the optimized and X-ray structures. However, our findings reported in the previous section indicate that it is sufficient to consider only optimized geometries to elucidate the influence of the basis set on $\beta$. We have calculated the geometry of HONS starting from nonplanar $C_{1}$ symmetry as well as constrained $C_{s}$ planar symmetry with trans and cis-orientation of the hydroxy group. The results (see Ref. 53 for details) show nearly identical hyperpolarizabilities and a small difference in energies $(<0.5 \mathrm{kcal} / \mathrm{mol})$ for considered conformations. Therefore, for all the other calculations, the geometry optimization was carried out assuming the planar structure of the $C_{S}$ symmetry with the trans orientation of the $\mathrm{OH}$ group as being the global minimum.

The changes in $\beta$ 's with the extension of the basis set from the simplest 6-31G to three split valence with polarization and diffuse functions demonstrate the same trends found for $p$-NA (Fig. 1 and Tables V and VI in Ref. 53). The most pronounced increase/decrease in hyperpolarizability is ob- served when first sets of diffuse and polarization functions are added (19\% and 24\%). Addition of $d$-functions also leads to a decrease in conjugation, which is reflected in the changes in the bond length distributions and the decrease in the dipole moment. The addition of the second set of $d$-functions leads to $5 \%$ decrease in $\beta$. The augmentation of 6-31G with diffuse functions results in negligible changes in the geometry and a small increase in the dipole moment. This is in agreement with the results on $p$-NA from the previous section. Again, in agreement with $p$-NA, the addition of polarization and diffuse functions on hydrogen atoms changes neither geometry nor hyperpolarizability. The addition of the $f$-functions and extension to the triple-zeta basis set leads to a small decrease in $\beta$.

The results obtained for HONS with other DFT functionals as well as with the HF method show the same trends in hyperpolarizabilities as reported in the previous section. PBE0 and BMK give $6 \%$ and $21 \%$ lower values, respectively, while B3LYP yields a 10\% higher value. The HF method significantly underestimates hyperpolarizability even more so than for $p$-NA. The multiplicative dispersion correction $(\Delta=1.58)$ is found to be $18 \%$ higher than that for $p$-NA. This is expected because the stilbene $\pi$-bridge is more polarizable than the benzene one and is, therefore, characterized by more pronounced susceptibility to any changes.

One can see from Fig. 1 that the relative changes from one basis set to another are nearly the same for $p$-NA and HONS (both less than 5\%). One can conclude that for D/A benzenes, stilbenes, and probably all other conjugated organic chromophores, it is sufficient to carry out calculations with a smaller basis set, followed by an appropriate rescaling. From our results, one may also conclude that the qualitative picture of variation of the geometry, dipole moment, hyperpolarizability with changes in the basis set for HONS is the same as for $p$-NA.

The relative increase in $\beta$ from $p$-NA to HONS [at static limit with the $6-31+\mathrm{G}(d)$ basis set] is equal to $3.7(3.3)$, 8.3(8.0), 7.9(7.6), 8.3(8.1), 6.9(6.4), 5.3(4.5) for HF, B97-2, PBE0, B3LYP, BMK, and MP2, respectively (values in parenthesis correspond to planar $C_{2 v}$ structure of $p$-NA). 
TABLE II. Dependence of $\beta(2 \omega, \omega, \omega)$ [obtained with the HF multiplicative factor ( $\Delta=1.33)$; given in a.u.] on a solvent for different DFT functionals, MP2 and CCSD methods in comparison with experimental data (from Ref. 28 obtained at $\lambda=1064 \mathrm{~nm}$ ).

\begin{tabular}{|c|c|c|c|c|c|c|c|c|c|c|c|c|c|c|}
\hline \multirow[b]{2}{*}{ Solvent } & \multirow[b]{2}{*}{ Expt. } & \multicolumn{7}{|c|}{$6-31+\mathrm{G}(d)$} & \multicolumn{6}{|c|}{$6-311+\mathrm{G}(2 d f, p d)$} \\
\hline & & B97-2 & PBE0 & B3LYP & BMK & $\mathrm{HF}$ & MP2 & CCSD & B97-2 & PBE0 & B3LYP & BMK & $\mathrm{HF}$ & MP2 \\
\hline $\begin{array}{l}\text { Gas- } \\
\text { phase }\end{array}$ & 1787 & 2201 & 2089 & 2320 & 2070 & 964 & 2294 & 1902 & 1887 & 1835 & 2059 & 1817 & 843 & 1940 \\
\hline$p$-dioxane & $4530-4694$ & 4329 & 4289 & 4741 & 4365 & 1967 & 4851 & $\cdots$ & $\cdots$ & $\cdots$ & $\cdots$ & $\cdots$ & $\cdots$ & $\cdots$ \\
\hline $\mathrm{CHCl}_{3}$ & 4950 & 6830 & 6823 & 7453 & 7056 & 3139 & 8031 & $\cdots$ & $\cdots$ & $\cdots$ & $\cdots$ & $\cdots$ & $\cdots$ & $\cdots$ \\
\hline Acetone & 7195 & 9789 & 9817 & 10550 & 10244 & 4620 & 12306 & $\cdots$ & $\cdots$ & $\cdots$ & $\cdots$ & $\cdots$ & $\cdots$ & $\cdots$ \\
\hline $\mathrm{CH}_{3} \mathrm{CN}$ & 8320 & 10321 & 10365 & 11113 & 10842 & 4912 & 13174 & $\cdots$ & $\cdots$ & $\cdots$ & $\cdots$ & $\cdots$ & $\cdots$ & $\cdots$ \\
\hline Methanol & $8890-10186$ & 10233 & 10274 & 11020 & 10744 & 4862 & 13029 & $\cdots$ & $\cdots$ & $\cdots$ & $\cdots$ & $\cdots$ & $\cdots$ & $\cdots$ \\
\hline
\end{tabular}

Among DFT methods, BMK yields the lowest increase upon elongation of the $\pi$-system, which is still somewhat higher than that of MP2 method. The other three exchangecorrelation functionals give nearly the same results. The ratio obtained at the HF level is significantly smaller than all the other ratios. In the next section, we present a more rigorous comparison, which takes into account both solvation effects and the frequency dependence.

\section{Comparison of the results: Solvent and frequency dependence}

To determine the reliability of the methods considered in this work, we have compared our results with the most accurate experimental values available in the literature. For a $p$-NA molecule, there is an experimental gas-phase value as well as results for different solvents. These data were recently analyzed and presented in the unified scale by Reis, ${ }^{28}$ who used the most recent and reliable value of $\beta$ of liquid $\mathrm{CCl}_{4}$ (from Ref. 62) as a benchmark. This benchmark value uses the experimental data from Refs. 63 and is calibrated against the accurately known theoretical predictions of the third harmonic generation for helium. We believe that the set of these experimental data (presented in the Taylor convention) is the most accurate for comparison with our theoretical predictions.

In Table II, we have compared hyperpolarizabilities of $p$-NA calculated in different solvents. The B97-2, B3LYP, and PBE0 functionals behave in nearly the same manner. B3LYP gives systematically higher values than B97-2, while PBE0 results show somewhat more rapid increase with the change in the solvent polarity.

Among GGA functionals, PBE0 shows the best agreement with the experiment (maximum error does not exceed $35 \%)$. The BMK functional provides the smallest value for an isolated $p$-NA molecule while it leads to the highest hyperpolarizability upon solvation. Maximum error $(\sim 42 \%)$ is found for three solvents (except 1,4-dioxane and methanol), while BMK shows the best agreement among all DFT methods for isolated (gas-phase) molecule. The results obtained at the MP2 level appear to be higher by factor $\sim 1.5$ (the maximum error is $71 \%$ for acetone) for both isolated and solvated molecule, while the HF method leads to a systematic underestimation of experimental hyperpolarizabilities (the maximum error is $41 \%$ for acetonitrile).
In spite of some differences in comparison of the absolute values, the relative increase in $\beta$ upon solvation is well reproduced by DFT. Comparison of the ratios of $\beta$ 's in the polar solvent $\mathrm{CH}_{3} \mathrm{CN}$ (methanol is not taken because of a possible $\mathrm{H}$-bonds formation, which complicates the comparison) to $\beta$ 's in the gas phase shows the following values: 4.7, 4.7, 5.0, 4.8, 5.2, 5.1, and 5.7 for experimental data, B97-2, PBE0, B3LYP, BMK, HF, and MP2, respectively. The HF method also agrees well with experimental trends, while the MP2 method somewhat overestimates the solvent dependence of $\beta$ for $p$-NA. The most significant conclusion is that the polarizable continuum model appears to describe the solvent effect successfully.

The gas phase values of $\beta$ predicted with both 6-31 $+\mathrm{G}(d)$ and $6-311+\mathrm{G}(2 d f, p d)$ basis sets are compared to the experimental one on Table II. The best agreement is found for the CCSD method. It overestimates the experiment by $6.4 \%$ only. Hyperpolarizability values predicted using the larger basis set are in better agreement with experiment, except for the HF method, which underestimates hyperpolarizability even more. However, our recommendation to use the $6-31+\mathrm{G}(d)$ basis set still holds because the relative trends in $\beta$ values upon variation of the basis set are rather similar and the relative changes in hyperpolarizability for short and long $\pi$-systems ( $p$-NA and HONS, respectively) do not change significantly.

In order to access the dynamic hyperpolarizability values directly, we applied SOS approach in conjunction with the aposteriori Tamm-Dancoff approximation (ATDA) ${ }^{46}$ to estimate the properties of the excited states. The results are summarized in Table III. The static SOS hyperpolarizabilities (second line) agree well with the results of FF calculations, collected in the top line. When 24 states are taken into account in the SOS formula, the values are only $2 \%-10 \%$ higher than the respective FF values. The frequency correction $\Delta$ calculated at HF level is less than $10 \%$ below the CPHF value for dynamic hyperpolarizability, presented in the last column for comparison. However, the SOS/ATDADFT values for the frequency correction appear to be significantly higher than the CPHF value used to predict frequency dependent hyperpolarizability reported in Table II. One can see that the agreement in the absolute values with experiment is a result of an error cancellation between the overestimated DFT static hyperpolarizability and underestimated CPHF dispersion correction values. However, the relative changes 
TABLE III. Static and dynamic $(\lambda=1064 \mathrm{~nm})$ hyperpolarizabilities of $p$-NA predicted by FF and TD/SOS methods with the $6-31+G^{*}$ basis set. The values calculated using the analytical derivatives with the CPHF method are reported in the last column.

\begin{tabular}{lcccccc}
\hline \hline & B97-2 & PBE0 & B3LYP & BMK & HF & CPHF \\
\hline$\beta(0), \mathrm{FF}$ & 1596 & 1571 & 1745 & 1557 & 726 & 726 \\
$\beta(0), \mathrm{SOS}$ & 1735 & 1661 & 1938 & 1537 & 687 & \\
$\beta(2 \omega, \omega, \omega)$ & 2940 & 2754 & 3433 & 2409 & 863 & 964 \\
$\Delta, \mathrm{FF}$ & 1.69 & 1.66 & 1.77 & 1.57 & 1.26 & 1.33 \\
\hline \hline
\end{tabular}

in $\beta$ 's with the molecular size would remain the same. Therefore, the use of Eq. (4) improves only the absolute values of $\beta$ 's.

As we have mentioned, unequivocal experimental values are available for $p$-NA only. It means that the only way for comparison of the calculated results for the set of molecules presented in Scheme 1 with experiment would be comparison of ratios (a relative increase/decrease in $\beta$ for a given molecule to $\beta$ of $p$-NA). This technique results in the elimination of ambiguities. Thus, it should be expected that the ratios of $\beta$ 's for two given molecules measured with the same method and at the same conditions would be nearly the same. Incidentally, the relative values rather than absolute values of hyperpolarizability is of importance in the rational molecular design of effective NLO materials. In Table IV, we present the comparison of the relative changes in hyperpolarizability for D/A-substituted benzenes and stilbenes in planar geometry. Experimental data were taken from Ref. 29 so that two comparable molecules (D/A substituted benzene and D/A-substituted stilbene) are studied under the same conditions and in the same laboratory.

One can see from Table IV that all DFT methods overestimate the increase in hyperpolarizability from the benzene to stilbene $\pi$-system. BMK is the most accurate of all considered functionals; its overestimation is 1.5-2.0. Among the three GGA functionals utilized, PBE0 seems to perform better than others.
Unfortunately, even the ratios of hyperpolarizabilities vary from one reference to another. For instance, in columns 2 and 3 of Table IV the ratio of $\beta_{\text {MONS }} / \beta_{\text {HONS }}$ would be 1.13 in weakly polar $\mathrm{CHCl}_{3}$, while from the last two columns, this ratio is 1.65 in nonpolar $p$-dioxane. In Ref. 67 the ratio of $\mu \beta$ product for MONS and MONB is equal to 15.3 while a nearly twice lower value was obtained in Ref. 66 . We use the latter for comparison because it seems to be more consistent with the other data. If we consider the ratio of $\beta_{\text {DANS }} / \beta_{\text {DACS }}$ $(4200 / 820=5.1$; the fourth and fifth columns), then it would appears that our DFT calculations underestimate this ratio. Also from our results it follows that the higher the value is of $\beta$, the more it is overestimated by DFT methods. However, GGA functionals overestimate the ratio of $\mu \beta_{\text {DANS }} / \mu \beta_{\text {DANB }}$ nearly twice, while for molecules with weaker substituents (HONS/HONB and MONS/MONB) they demonstrate even a larger (nearly threefold) overestimation instead of expected smaller one. The results obtained at the HF level show a significant underestimation for the first four molecular pairs (columns 2-5) while experimental ratios in the last two columns are overestimated by HF. It seems that one can find a pair of molecules with the measured hyperpolarizability ratio that matches the results of any given quantum chemical method. In part, this variety may be explained by specific solvation effects (i.e., the formation of H-bonded associates) that affect hyperpolarizability, but are not taken into account in our calculations.

TABLE IV. Comparison of calculated and experimental ratios of $\beta(2 \omega, \omega, \omega)_{(\mathrm{D} / \mathrm{A} \text {-stilbene) }} / \beta(2 \omega, \omega, \omega)_{\text {(D/A-benzene) }}$.

\begin{tabular}{|c|c|c|c|c|c|c|}
\hline & MONS / $p$ NA & $\mathrm{HONS} / p \mathrm{NA}$ & DACS/DACB & DANS/DANB & MONS/MONB & HONS/HONB \\
\hline Expt. & $105 / 23=4.6^{\mathrm{a}}$ & $93 / 23=4.1^{\mathrm{a}}$ & & & $28 / 5.1=5.5^{\mathrm{b}}$ & $17 / 3.0=5.7^{\mathrm{b}}$ \\
\hline$\left(\beta \times 10^{-30} \mathrm{esu}\right)$ & $\lambda=1064, \mathrm{CHCl}_{3}$ & $\lambda=1064, \mathrm{CHCl}_{3}$ & & & $\lambda=1064, p$-dioxane & $\lambda=1064, p$-dioxane \\
\hline B97-2 & $657 / 59.1=11.1$ & $623 / 59.1=10.5$ & & & $423 / 27.5=15.4$ & $382 / 22.3=17.1$ \\
\hline PBE0 & $596 / 58.9=10.1$ & $572 / 58.9=9.7$ & & & $390 / 26.6=14.7$ & $356 / 21.8=16.3$ \\
\hline B3LYP & $762 / 64.4=11.8$ & $719 / 64.4=11.2$ & & & $480 / 30.5=15.7$ & $433 / 24.7=17.5$ \\
\hline BMK & $469 / 60.9=7.7$ & $452 / 60.9=7.4$ & & & $313 / 25.0=12.5$ & $288 / 20.9=13.8$ \\
\hline $\mathrm{HF}$ & $111 / 32.2=3.4$ & $110 / 32.2=3.4$ & & & $85 / 10.3=8.3$ & $81 / 9.0=9.0$ \\
\hline MP2 & $332 / 81.1=4.1$ & $311 / 81.1=3.8$ & & & $232 / 27.2=8.5$ & $226 / 23.0=9.8$ \\
\hline Expt. & & & $820 / 94=8.7^{\mathrm{c}}$ & $4200 / 355=11.8^{\mathrm{c}}$ & $450 / 55=8.2^{\mathrm{d}}$ & \\
\hline$\left(\mu \beta \times 10^{-48}\right.$ esu $)$ & & & $\lambda=1890$, DMSO & $\lambda=1890$, DMSO & $\lambda=1064, \mathrm{CHCl}_{3}$ & \\
\hline B97-2 & & & $7377 / 424=17.4$ & $27598 / 1238=22.3$ & $6772 / 310=21.8$ & \\
\hline PBE0 & & & $6818 / 416=16.4$ & $24551 / 1217=20.2$ & $6017 / 296=20.3$ & \\
\hline B3LYP & & & $7575 / 440=17.2$ & $34018 / 1382=24.6$ & $8144 / 356=22.9$ & \\
\hline BMK & & & $5096 / 382=13.3$ & $18762 / 1293=14.5$ & $4721 / 296=15.9$ & \\
\hline $\mathrm{HF}$ & & & $1257 / 226=5.6$ & $2090 / 519=4.0$ & $960 / 107=9.0$ & \\
\hline MP2 & & & $\ldots$ & $\ldots$ & $2619 / 267=9.8$ & \\
\hline
\end{tabular}

${ }^{\mathrm{a}}$ Reference 41.

${ }^{\mathrm{b}}$ Reference 64.

${ }^{\mathrm{c}}$ Reference 65.

${ }^{\mathrm{d}}$ Reference 66. 
The values obtained at the MP2 level of theory appear to be in better agreement with the experimental data in general. However, the difference between MP2 and BMK ratios is rather small. For pairs MONS/ $p$-NA, HONS / $p$-NA, MONS/ $\mathrm{MONB}$, and $\mathrm{HONS} / \mathrm{HONB}$ the BMK/MP2 ratios are equal to $1.88,1.93,1.47$, and 1.41 , correspondingly. Thus, BMK yields an overestimation of MP2 results by a factor of 1.4 1.9, which can be used to scale BMK results for other $\mathrm{D}-\pi$-A molecules with the stilbene $\pi$-system. A similar rescaling procedure can be done for other functionals. Before frequency and solvent dependence is taken into account, the BMK/MP2 ratios are even more uniform $(1.42,1.42,1.26$, 1.27) for the pairs of stilbenelike systems studied.

\section{CONCLUSIONS}

This study answers four essential questions in the rational design of NLO materials: (1) the choice of the method, (2) the choice of the basis set, (3) the effects of molecular planarity, and (4) the best technique to compare the calculated results to each other and to experiment. Our calculations show that the results obtained with the 6-31G basis set are nearly identical to those calculated with the $6-31+\mathrm{G}^{*}$ basis, in agreement with observation by Masunov and Tretiak. ${ }^{26}$ However, a detailed analysis demonstrates that this originates from the error cancellation. While the use of polarization functions leads to the interatomic charge redistribution, the use of diffuse functions results in the predominantly intra-atomic charge redistribution. We found the addition of one set of polarization and diffuse functions sufficient and recommend the use of the $6-31+\mathrm{G}^{*}$ basis set to obtain the hyperpolarizability ratios in the series of molecules. We recommend the smaller $6-31 \mathrm{G}^{*}$ basis set for description of the ground state geometry. A further extension of the basis allows one to obtain more accurate absolute hyperpolarizabilities, while relative values remain nearly the same.

We also found that the planar geometry constraints do not significantly affect molecular hyperpolarizabilities. Most NLO-active molecules are $\pi$-conjugated, and their structures are usually planar. In some cases, planarity can be distorted by pyramidalization of the amino groups, rotation of the alcoxy groups, or steric repulsion of hydrogen atoms across the $\pi$-conjugated bridge. In these or similar cases, planar geometry constraints can be applied, which can increase the absolute value of $\beta$ up to $10 \%$ but does not affect the relative hyperpolarizabilities.

The most significant question is the choice of the appropriate method for calculation. We have found that HF underestimates both absolute values of hyperpolarizability and the relative increase/decrease in $\beta$ in the series of molecules. At the same time, DFT methods were found to be more accurate without a significant computational overhead. A fast development of new and improved exchange-correlation functionals resulted in the hybrid meta-GGA functional BMK, which we found to overestimate the hyperpolarizability to the lesser extent than the other functionals. We expect the functionals with a larger fraction of the HF exchange to perform even better. Among the three hybrid GGA functionals that do not explicitly include the electron kinetic energy, PBE0 seems to perform slightly better than others. MP2 results are found to be more accurate than those obtained with DFT methods. However, even the MP2 level of theory does not appear to be perfect. The best agreement with experiment is found for the HONS / $p$-NA molecular pair ( $8 \%$ underestimation) while the worst is for the HONS/HONB molecular pair $(72 \%$ overestimation). However, the difference in the relative values of $\beta$ for DFT and MP2 does not vary significantly. We argue that the use of an appropriate scaling factor for DFT functionals will improve the accuracy.

The choice of the appropriate method is closely related to the problem of comparison of the results, effects of the solvent, and frequency dependence. We argue that comparison of absolute values of molecular hyperpolarizabilities is not very meaningful due to systematic errors in the experimental data. Comparison of the ratios for molecules studied in the same laboratory can compensate the ambiguities in conventions and standards used by different experimental groups. However, comparison of the calculated values with the experimental ones remains somewhat ambiguous even though conventions and standards are taken into account in an appropriate way. Since the main goal of calculations is to select more efficient molecules from a particular series, a semiquantitative level of accuracy in the prediction of molecular NLO properties should be sufficient.

We found that the error due to neglect of the solvent and frequency effects can be in the 15\%-45\% range, which is comparable with the uncertainty in experimental data. Therefore, the solvent and frequency dependence may be neglected. The static gas-phase hyperpolarizability ratio of BMK to MP2 is nearly constant for different molecular pairs (D/A-benzene/D/A-stilbene). Therefore, we can suggest to scale BMK hyperpolarizabilities for stilbenelike D- $\pi$-A molecules by 1.35 to obtain the MP2 accuracy at a low computational cost. The other DFT methods can also be used with an appropriate scaling.

Finally, we would like to reiterate the great need in the development of more accurate DFT functionals, as well as databases of consistent experimental data, which can be used for benchmarking purposes. Studies along these lines are underway.

\section{ACKNOWLEDGMENTS}

This work was supported, in part, by the National Science Foundation under Grant No. CCF 0740344. The authors are thankful to DOE NERSC and UCF I2Lab for the generous donation of computer time. S.T. gratefully acknowledges support by the UCF NSTC and UCF Graduate Studies Office through the Summer Mentoring Research Fellowship.

\footnotetext{
${ }^{1}$ J. Zyss, in Molecular Nonlinear Optics: Materials, Physics and Devices (Academic, New York, 1994); H. S. Nalwa and S. Miyata, in Nonlinear Optics of Organic Molecules and Polymers (CRC, Boca Raton, 1997); H. S. Nalwa, in Handbook of Advanced Electronic and Photonic Materials and Devices. Nonlinear Optical Materials (Academic, San Diego, 2000), Vol. 9; F. Wurthner, R. Wortmann, and K. Meerholz, ChemPhysChem 3, 17 (2002); M. G. Papadopoulos, in Nonlinear Optical Responses of Molecules, Solids and Liquids: Methods and Applications (Research Signpost, Trivandrum, 2003); S. R. Marder, Chem. Commun. (Cambridge) 2006, 131

${ }^{2}$ M. Torrent-Sucarrat, M. Sola, M. Duran, J. M. Luis, and B. Kirtman, J.
} 
Chem. Phys. 118, 711 (2003)

${ }^{3}$ B. Skwara, W. Bartkowiak, A. Zawada, R. W. Gora, and J. Leszczynski, Chem. Phys. Lett. 436, 116 (2007).

${ }^{4}$ D. P. Shelton and J. E. Rice, Chem. Rev. (Washington, D.C.) 94, 3 (1994)

${ }^{5}$ K. Y. Suponitsky, T. V. Timofeeva, and M. Y. Antipin, Russ. Chem. Rev. 75, 457 (2006)

${ }^{6}$ W. Koch and M. C. Holthausen, A Chemist's Guide to Density Functional Theory, 2nd ed. (Wiley-VCH, New York, 2002); M. Springborg, in Chemical Modelling: Applications and Theory, edited by A. Hinchliffe (Royal Society of Chemistry, Cambridge, 2004), Vol. 9, p. 69.

${ }^{7}$ B. Champagne, E. A. Perpete, S. J. A. van Gisbergen, E. J. Baerends, J. G. Snijders, C. Soubra-Ghaoui, K. A. Robins, and B. Kirtman, J. Chem. Phys. 109, 10489 (1998).

${ }^{8}$ B. Champagne, E. A. Perpete, D. Jacquemin, S. J. A. van Gisbergen, E. J. Baerends, C. Soubra-Ghaoui, K. A. Robins, and B. Kirtman, J. Phys. Chem. A 104, 4755 (2000).

${ }^{9}$ M. Gruning, O. V. Gritsenko, S. J. A. van Gisbergen, and E. J. Baerends, J. Chem. Phys. 116, 9591 (2002).

${ }^{10}$ M. Gruning, O. V. Gritsenko, S. J. A. van Gisbergen, and E. J. Baerends, J. Chem. Phys. 114, 652 (2001).

${ }^{11}$ L. Jensen and P. T. van Duijnen, J. Chem. Phys. 123, 074307 (2005).

${ }^{12}$ K. D. Singer, J. E. Sohn, L. A. King, H. M. Gordon, H. E. Katz, and C. W. Dirk, J. Opt. Soc. Am. B 6, 1339 (1989).

${ }^{13}$ E. R. Davidson, B. E. Eichinger, and B. H. Robinson, Opt. Mater. (Amsterdam, Neth.) 29, 360 (2006); C. M. Isborn, A. Leclercq, F. D. Vila, L. R. Dalton, J. L. Bredas, B. E. Eichinger, and B. H. Robinson, J. Phys. Chem. A 111, 1319 (2007).

${ }^{14}$ T. H. Dunning, J. Chem. Phys. 90, 1007 (1989); R. A. Kendall, T. H. Dunning, and R. J. Harrison, ibid. 96, 6796 (1992); D. E. Woon and T. H. Dunning, ibid. 98, 1358 (1993); 100, 2975 (1994); A. K. Wilson, T. vanMourik, and T. H. Dunning, J. Mol. Struct.: THEOCHEM 388, 339 (1996).

${ }^{15}$ F. Jensen, J. Chem. Phys. 115, 9113 (2001); 116, 7372 (2002); 117, 9234 (2002); 118, 2459 (2003).

${ }^{16}$ D. L. P. Chong, M. Gruning, and E. J. Baerends, J. Comput. Chem. 24, 1582 (2003).

${ }^{17}$ G. D. Zeiss, W. R. Scott, N. Suzuki, D. P. Chong, and S. R. Langhoff, Mol. Phys. 37, 1543 (1979).

${ }^{18}$ L. Jensen, P. T. van Duijnen, J. G. Snijders, and D. P. Chong, Chem. Phys. Lett. 359, 524 (2002).

${ }^{19}$ A. J. Sadlej, Collect. Czech. Chem. Commun. 53, 1995 (1988); A. J. Sadlej, Theor. Chim. Acta 79, 123 (1991); 81, 45 (1991); 81, 339 (1992); I. Cernusak, V. Kello, and A. J. Sadlej, Collect. Czech. Chem. Commun. 68, 211 (2003)

${ }^{20}$ B. O. Roos and A. J. Sadlej, Chem. Phys. 94, 43 (1985).

${ }^{21}$ H. Sekino and R. J. Bartlett, J. Chem. Phys. 98, 3022 (1993).

${ }^{22}$ P. Rozyczko and R. J. Bartlett, J. Chem. Phys. 107, 10823 (1997).

${ }^{23}$ T. Pluta and A. J. Sadlej, Chem. Phys. Lett. 297, 391 (1998).

${ }^{24}$ T. Pluta and A. J. Sadlej, J. Chem. Phys. 114, 136 (2001).

${ }^{25}$ Z. Benkova, A. J. Sadlej, R. E. Oakes, and S. E. J. Bell, J. Comput. Chem. 26, 145 (2005); Z. Benkova, A. J. Sadlej, R. E. Oakes, and S. E. J. Bell, Theor. Chem. Acc. 113, 238 (2005).

${ }^{26}$ A. M. Masunov and S. Tretiak, J. Phys. Chem. B 108, 899 (2004).

${ }^{27}$ A. Willetts, J. E. Rice, D. M. Burland, and D. P. Shelton, J. Chem. Phys. 97, 7590 (1992); R. F. Shi and A. F. Garito, in Characterization Techniques and Tabulations for Organic Nonlinear Optical Materials, edited by M. G. Kuzyk and C. W. Dirk (Dekker, New York, 1998), p. 1.

${ }^{28}$ H. Reis, J. Chem. Phys. 125, 014506 (2006).

${ }^{29}$ K. D. Singer, S. F. Hubbard, A. Schober, L. M. Hayden, and K. Johnson, in Characterization Techniques and Tabulations for Organic Nonlinear Optical Materials, edited by M. G. Kuzyk and C. W. Dirk (Dekker, New York, 1998), p. 311

${ }^{30}$ P. Salek, T. Helgaker, O. Vahtras, H. Agren, D. Jonsson, and J. Gauss, Mol. Phys. 103, 439 (2005); P. Salek, O. Vahtras, T. Helgaker, and H. Agren, J. Chem. Phys. 117, 9630 (2002).

${ }^{31}$ G. C. Yang, S. Q. Shi, W. Guan, L. Fang, and Z. M. Su, J. Mol. Struct.: THEOCHEM 773, 9 (2006).

${ }^{32}$ H. Soscun, O. Castellano, Y. Bermudez, C. Toro, N. Cubillan, A. Hinchliffe, and X. N. Phu, Int. J. Quantum Chem. 106, 1130 (2006).

${ }^{33}$ C. K. Wang, Y. H. Wang, Y. Su, and Y. Luo, J. Chem. Phys. 119, 4409 (2003).
${ }^{34}$ L. Frediani, H. Agren, L. Ferrighi, and K. Ruud, J. Chem. Phys. 123, 144117 (2005).

${ }^{35}$ M. Y. Balakina and S. E. Nefediev, Int. J. Quantum Chem. 106, 2245 (2006).

${ }^{36}$ L. C. T. Shoute, R. Helburn, and A. M. Kelley, J. Phys. Chem. A 111, 1251 (2007)

${ }^{37}$ H. D. Cohen and C. C. Roothaan, J. Chem. Phys. 43, S34 (1965); C. E. Dykstra and P. G. Jasien, Chem. Phys. Lett. 109, 388 (1984); H. Sekino and R. J. Bartlett, J. Chem. Phys. 85, 976 (1986); S. P. Karna and M. Dupuis, J. Comput. Chem. 12, 487 (1991).

${ }^{38}$ J. F. Ward, Rev. Mod. Phys. 37, 1 (1965); B. J. Orr and J. F. Ward, Mol. Phys. 20, 513 (1971)

${ }^{39}$ P. Kaatz, E. A. Donley, and D. P. Shelton, J. Chem. Phys. 108, 849 (1998).

${ }^{40}$ K. Y. Suponitsky, K. A. Lyssenko, and M. Y. Antipin (unpublished).

${ }^{41}$ K. Clays and A. Persoons, Phys. Rev. Lett. 66, 2980 (1991); K. Clays and A. Persoons, Rev. Sci. Instrum. 63, 3285 (1992).

${ }^{42}$ M. Muthuraman, R. Masse, J. F. Nicoud, and G. R. Desiraju, Chem. Mater. 13, 1473 (2001)

${ }^{43}$ M. J. Frisch, G. W. Trucks, H. B. Schlegel et al., Gaussiano3, Revision D.01 (Gaussian, Inc., Wallingford, CT, 2004).

${ }^{44}$ D. Jacquemin, B. Champagne, and C. Hattig, Chem. Phys. Lett. 319, 327 (2000); L. Sanguinet, J. L. Pozzo, M. Guillaume, B. T. Champagne, F. Castet, L. Ducasse, E. Maury, J. Soulie, F. Mancois, F. Adamietz, and V. Rodriguez, J. Phys. Chem. B 110, 10672 (2006).

${ }^{45}$ Y. Luo, H. Agren, and S. Stafstrom, J. Phys. Chem. 98, 7782 (1994).

${ }^{46}$ I. A. Mikhailov, S. Tafur, and A. E. Masunov, Phys. Rev. A 77, 012510 (2008).

${ }^{47}$ F. A. Hamprecht, A. J. Cohen, D. J. Tozer, and N. C. Handy, J. Chem. Phys. 109, 6264 (1998).

${ }^{48}$ A. D. Becke, Phys. Rev. A 38, 3098 (1988); C. T. Lee, W. T. Yang, and R. G. Parr, Phys. Rev. B 37, 785 (1988).

${ }^{49}$ C. Adamo and V. Barone, J. Chem. Phys. 110, 6158 (1999).

${ }^{50}$ A. D. Boese and J. M. L. Martin, J. Chem. Phys. 121, 3405 (2004).

${ }^{51}$ A. J. Cohen, N. C. Handy, and D. J. Tozer, Chem. Phys. Lett. 303, 391 (1999).

${ }^{52}$ S. Tafur, K. D. Belfield, and A. E. Masunov (unpublished).

${ }^{53}$ See EPAPS Document No. E-JCPSA6-128-001823 for five pages containing detailed information on basis set dependence of hyperpolarizability and geometry characteristics for $p$-NA and HONS for all methods considered in the present study. For more information on EPAPS, see http://www.aip.org/pubservs/epaps.html.

${ }^{54}$ H. Reis, A. Grzybowski, and M. G. Papadopoulos, J. Phys. Chem. A 109, 10106 (2005)

${ }^{55}$ A. N. Rashid, J. Mol. Struct.: THEOCHEM 681, 57 (2004).

${ }^{56}$ A. Rauk, L. C. Allen, and E. Clementi, J. Chem. Phys. 52, 4133 (1970); C. W. Bock, P. George, and M. Trachtman, Theor. Chim. Acta 69, 235 (1986); O. Bludsky, J. Sponer, J. Leszczynski, V. Spirko, and P. Hobza, J. Chem. Phys. 105, 11042 (1996).

${ }^{57}$ A. Masunov and J. J. Dannenberg, J. Phys. Chem. A 103, 178 (1999).

${ }^{58}$ K. Kaufmann, C. Nager, and M. Jungen, Chem. Phys. 95, 385 (1985).

${ }^{59}$ T. Troxler, M. R. Topp, B. S. Metzger, and L. H. Spangler, Chem. Phys Lett. 238, 313 (1995); S. S. Siewert and L. H. Spangler, J. Phys. Chem. 99, 9316 (1995); B. S. Metzger and L. H. Spangler, J. Phys. Chem. A 101, 5431 (1997).

${ }^{60}$ L. H. Spangler, R. Vanzee, and T. S. Zwier, J. Phys. Chem. 91, 2782 (1987); L. H. Spangler, R. D. Vanzee, S. C. Blankespoor, and T. S. Zwier, ibid. 91, 6077 (1987); B. B. Champagne, J. F. Pfanstiel, D. F. Plusquellic, D. W. Pratt, W. M. Vanherpen, and W. L. Meerts, ibid. 94, 6 (1990).

${ }^{61}$ R. Daum, T. Hansson, R. Norenberg, D. Schwarzer, and J. Schroeder, Chem. Phys. Lett. 246, 607 (1995).

${ }^{62}$ R. D. Pyatt and D. P. Shelton, J. Chem. Phys. 114, 9938 (2001).

${ }^{63}$ P. Kaatz and D. P. Shelton, J. Chem. Phys. 105, 3918 (1996).

${ }^{64}$ L. T. Cheng, W. Tam, S. H. Stevenson, G. R. Meredith, G. Rikken, and S. R. Marder, J. Phys. Chem. 95, 10631 (1991).

${ }^{65}$ A. Dulcic and C. Sauteret, J. Chem. Phys. 69, 3453 (1978); A. Dulcic, C. Flytzanis, C. L. Tang, D. Pepin, M. Fetizon, and Y. Hoppilliard, J. Chem. Phys. 74, 1559 (1981).

${ }^{66}$ E. Kelderman, W. A. J. Starmans, J. P. M. Vanduynhoven, W. Verboom, J. F. J. Engbersen, D. N. Reinhoudt, L. Derhaeg, T. Verbiest, K. Clays, and A. Persoons, Chem. Mater. 6, 412 (1994).

${ }^{67}$ R. A. Huijts and G. L. J. Hesselink, Chem. Phys. Lett. 156, 209 (1989). 